A. Weisbecker, M. Burmester \& A. Schmidt (Hrsg.): Mensch und Computer 2015 Workshopband, Stuttgart: Oldenbourg Wissenschaftsverlag, 2015, S. 291-295.

\title{
User Autonomy Protection in Mobile Coaching Systems
}

\author{
Joakim Haugen $^{1}$, Robbert Jan Beun ${ }^{2}$, Fiemke Griffioen-Both ${ }^{2}$ \\ Utrecht University, Master of Business Informatics, The Netherlands ${ }^{1}$ \\ Utrecht University, Dep. of Information and Computing Sciences, Interaction Technology, \\ The Netherlands ${ }^{2}$
}

\begin{abstract}
This paper presents preliminary findings from a study of user autonomy protection (UAP) in mobile coaching applications. Autonomy refers to doing things out of one's own volition, and is an important ethical principle in the health care sector. Protecting a patient's autonomy can significantly enhance treatment adherence and can have positive motivational effects. We are developing a mobile self-help app to be used by patients undergoing cognitive behavioral therapy for insomnia (CBT-I), and aim to implement UAP in template-based dialogues. Transcripts of real CBT-I sessions are studied in order to unearth specific speech acts that therapists use to protect the patient's autonomy in practice.
\end{abstract}

\section{Introduction}

The health care sector is currently moving towards an emphasis on patient autonomy (Legare et al., 2008). In contrast to the traditionally paternalistic approach of health care, in which health professionals more or less tell patients what to do, the new trend focuses on involving patients in making decisions about their own treatment. Autonomy refers to doing things out of one's own volition (Ryan \& Deci, 2000), and is considered a basic human need. It has gained recognition as an ethical principle in the health care sector. The trend towards patient autonomy is equally relevant to mobile coaching application design. Here, we suppose that a mobile coaching application is one that aids the user in attaining sustainable behavior change.

Supporting user autonomy protection (UAP) in mobile coaching systems can prove to have significant positive effects. According to the self-determination theory (SDT; Deci \& Ryan, 2000; Ryan \& Deci, 2000), autonomy is a key facilitator of intrinsic motivation. The more autonomous individuals feel in their behavior, the more intrinsically motivated they will be. These positive motivational effects can in turn lead to higher adherence to therapies and treatments. Lack of treatment adherence is a persistent problem in medical care (Vermeire et al., 2001), referring to a lack of compliance with the prescribed medical interventions of one's treatment plan (e.g. taking prescribed medication or performing prescribed activities). This 
may undermine the outcome of the treatment or diminish it entirely, depending on the nature of the treatment. This is clearly a problem, not only for the patient but also for the health care provider, as time and efforts spent on the treatment end up being wasted. The problem is exacerbated by the current bottleneck in health care provision.

Mobile coaching systems are built to support existing treatments or replace them entirely. These applications assist the user in performing various tasks and exercises. Mobile devices, particularly the smartphone, are an excellent platform for coaching systems for a variety of reasons. The particularly high market penetration of the device means that many patients already have the means to use the application. Furthermore, people usually bring their smartphones with them everywhere they go, so the devices are salient in their daily lives. Smartphones also contain a number of sensors that can provide real-time data input for applications. Because of these reasons, mobiles are not only highly practical, but also present an opportunity to influence the user in desirable ways. Persuasive technology aims to do just that (Fogg, 2002). Persuasive applications are designed to change the behavior of users, and do so by enhancing the user's motivation to perform the desired behavior or by making the behavior easier to do. Coaching systems can employ various persuasion strategies to increase the likelihood that the desired behavior is performed, leading to positive outcomes.

The present research studies the potential of UAP as a persuasive strategy. We are developing a mobile coaching system, SleepCare (Beun, 2013; Beun et al., 2014), to be used by patients undergoing cognitive behavioral therapy for insomnia (CBT-I). The app provides a number of tools such as an interactive sleep log, and provides consultation by means of template-based dialogue. We aim to implement UAP in the template-based dialogue of the app.

\section{Research goals and approach}

The goal of the present research is twofold. First, we aim to identify autonomy protection strategies. These are general principles that in some way protect the autonomy of the conversational partner. By taking the individual's need for autonomy into account, therapists can utilize specific speech acts that allow the client's autonomy to remain intact, or at least allow the infringement of the client's autonomy to be minimal. We expect autonomy protection to occur naturally and intuitively in therapist-client conversations, and we therefore distinguish these conversations as appropriate sources for the identification of autonomy protection strategies. The raw data used for the present research consists of audio recordings of several therapeutic conversations. More specifically, the recordings cover the therapy sessions of two different clients undergoing CBT-I. About 8 hours of conversational data has been transcribed, so as to serve as more appropriate objects of analysis. Speech acts related to UAP will be classified and subsequently generalized to a non domain-specific format that is generally applicable.

The analysis is partly grounded in theory, drawing upon existing literature (e.g. shared decision making in Charles et al, 1997; various forms of politeness in Brown \& Levinson, 1987) as hints of what could constitute autonomy protection strategies. The dialogue analysis therefore 
partly serves as confirmation. However, the findings of earlier work did not specifically address autonomy as a persuasive instrument, so they are viewed in a new light. Furthermore, the speech acts that protect an individual's autonomy have, to the best of our knowledge, not yet been documented.

The second aim of the present research is to implement the findings from the dialogue analysis in a mobile health care application. The SleepCare project was initiated in 2012 with the aim to develop a computerized coaching system for individuals undergoing CBT-I. The coaching system is being developed as a mobile app for Android smartphones, and it is intended to assist the user in various tasks and exercises. The app makes use of template-based dialogue to influence the user, and various persuasive strategies have already been applied to the current dialogue. We wish to increase the application's persuasive potential by also implementing UAP speech acts in the template-based dialogue of the app.

\section{Preliminary findings}

The present research project is currently in the dialogue analysis stage. At the time of writing, the transcripts have been examined in a global manner. Below, we outline the basic dialogue structure that the therapist [T] employs in order to assess treatment adherence:

- $\quad[\mathrm{T}]$ : Asks if a specific task / activity is going well

A. [C]: Confirms that the task is going well

- [T]: Gives positive feedback / compliment

B. [C]: Indicates that there is a problem or complication

- $\quad[\mathrm{T}]$ : Gives advice on how to tackle the problem

The primary purpose of each consultation session is to assess how pre-determined tasks and activities are going, identify potential problems and mitigate those. The structure above is a recurring pattern within each session. In the beginning of each session, [T] asks if there is anything [C] would like to discuss, essentially inviting [C] to indicate problem areas. [C] has a list of tasks, and for each one not already indicated as a problem area, [T] asks how it is going. The phrasing used here is interesting, because it is nearly always asked in such a way that [C]'s motivation to do the task is not questioned. For example, [T] might ask "Did you manage to...", rather than "Did you...", something we identify as facekeeping, a form of politeness aimed at maintaining [C]'s dignity. According to Fogg's model of behavior change (Fogg, 2009), the likelihood of an activity being performed is a negative exponential function of motivation and ability. The phrasing used by [T] does put [C]'s ability into question. We argue that phrasing that respects both motivation and ability is desirable.

The conversation may then branch out in one of two ways. [C] can confirm that there are no problems in performing the task, after which [T] usually inquires about the exact approach, which is subsequently outlined by [C]. When done, [T] gives positive feedback, usually in the form of a compliment (e.g. "What a creative idea!" or “That's a great way to do it!"). We identify such compliments as speech acts that conduce towards [C]'s feeling of competence. 
Strictly speaking, this is not a case of UAP. However, perceived competence is an important aspect of motivation. According to the cognitive evaluation theory (CET; Ryan \& Deci, 2000), the more competent an individual feels in performing a behavior, the more likely that behavior is to occur. CET argues that feelings of competence only enhance intrinsic motivation if accompanied by a sense of autonomy, so one could conclude that the inverse is true as well. In any case, we consider the reinforcement of perceived competence beneficial to treatment adherence.

The alternative route that the conversation might take is when $[\mathrm{C}]$ indicates having some kind of problem with performing the task. [T] then inquires about the problem and comes with suggestions as to how [C] can deal with the problem and still perform the task. Important to note is that [T] suggests various solutions, as opposed to ordering [C] to do something. We notice this in sentences like "You could try to...", "Maybe it's a good idea to...", and similar lines. [T] also doesn't attempt to make [C] promise to employ the suggested solution, but instead leaves it up to [C] to decide whether or not to employ the solution when the problem arises. We consider suggestion to be a UAP strategy.

Usually, [C] accepts the suggestions of [T]. However, it also occurs that a compromise has to be reached. For example, it is advisable that an insomnia patient avoids daytime naps so as to not decrease nighttime sleepiness. As such, [T] would recommend that [C] doesn't take any daytime naps. [C] might then argue that a daytime nap is needed because he/she feels a lack of energy otherwise. In our data, [C] indicate that he used to take a two hour nap every day around noon. [T] then argued that such a long nap decreases nighttime sleepiness, which is counter-productive. [T] recommended limiting the nap to 30 minutes, as that will have a less significant effect on the nighttime sleepiness, which [C] accepted. This was an instance of a UAP strategy that we define as negotiation, i.e. reaching a compromise between the ideal state (no naps at all) and the individual's preferred state (a two hour nap).

\section{Future work}

The preliminary findings of the dialogue analysis show promising signs that UAP is indeed applied in practice by therapists. The spectrum of potential UAP strategies shall be extended through detailed dialogue analysis. We call them potential because they need to be assessed in terms of theoretical correctness and their impact on user autonomy. Furthermore, each UAP speech act needs to be stripped down to a basic dialogue structure. The transcripts used for dialogue analysis were all based on CBT-I interventions, and findings can be applied directly to the SleepCare app. However, we expect UAP to be of great use to all kinds of coaching systems, so we will also discuss a general form of the dialogue structures of each UAP speech act.

\section{Acknowledgement}

This research is supported by Philips and Technology Foundation STW, Nationaal Initiatief Hersenen en Cognitie NIHC under the Partnership programme Healthy Lifestyle Solutions. 


\section{References}

Beun, R. J. (2013) Persuasive Strategies in Mobile Insomnia Therapy: Alignment, Adaptation, and Motivational support. Personal and Ubiquitous Computing 17(6), 1187-1195.

Beun, R. J., Griffioen-Both, F., Ahn, R., Fitrianie, S., \& Lancee, J. (2014) Modeling Interaction in Automated e-Coaching: A Case from Insomnia Therapy. 6th Int. Conf. on Advanced Cognitive Technologies and Applications. Venice, May 25-29, 2014.

Brown, P. \& Levinson, S. C. (1987) Politeness: Some universals in language usage (Vol. 4). Cambridge University Press.

Charles, C., Gafni, A. \& Whelan, T. (1997) Shared Decision-Making in Medical Encounter: What does it mean (or it takes two to tango). Soc. Sci. Med. 44(5), 681-692.

Deci, E. L., \& Ryan, R. M. (2000). The "what" and "why" of goal pursuits: Human needs and the selfdetermination of behavior. Psychological inquiry, 11(4), 227-268.

Fogg, B. J. (2002) Persuasive Technology: Using Computers to Change What We Think and Do. San Francisco: Morgan Kaufmann Publishers.

Fogg, B. J. (2009, April). A behavior model for persuasive design. InProceedings of the 4th international Conference on Persuasive Technology (p. 40). ACM.

Legare, F., Ratté, S., Gravel, K. \& Graham, I. D. (2008) Barriers and facilitators to implementing shared decision-making in clinical practice: Update of a systematic review of health professionals' perceptions. Pat. Educ. \& Counseling 73, 526-535.

Ryan, R. M. \& Deci, E. L. (2000). Intrinsic and extrinsic motivations: Classic definitions and new directions. Contemporary educational psychology, 25(1), 54-67.

Ryan, R. M. \& Deci, E. L. (2000) Self-determination theory and facilitation of intrinsic motivation, social development and well-being. Am Psychol 55(1), 68-78.

Vermeire, E, Hearnshaw, H, Van Royen, P \& Denekens, J (2001) Patient adherence to treatment: three decades of research. A comprehensive review. Journal of Clinical Pharmacy and Therapeutics 26, 331-342.

\section{Contact information}

Joakim Haugen: j.haugen@ @ students.uu.nl

Robbert Jan Beun: r.j.beun@uu.nl

Fiemke Griffioen-Both: f.griffioen-both@uu.nl 
